\title{
The Optimal Surface Roughness Condition on Diffusion Bonding
}

\author{
Hidetoshi Somekawa* and Kenji Higashi \\ Department of Metallurgy and Materials Science, Osaka Prefecture University, Sakai 599-8531, Japan
}

The surface roughness created by grit blast treatment affected diffusion bonding quality. The surface treatment is very important to remove the oxide films in combination with superplastic forming and diffusion bonding. Therefore, by using the superplastic magnesium alloys, the diffusion bonding tests carried out to obtain the relationship between the diffusion bonding quality and surface roughness. From the diffusion bonding results, in order to obtain high quality joining, the void shrinkage processes were not only diffusional controlled process but also plasticity controlled process. The optimal surface roughness was estimated equal to grain size.

(Received May 28, 2003; Accepted June 17, 2003)

Keywords: diffusion bonding, compressive lap shear strength, magnesium alloy, surface roughness, superplasticity

\section{Introduction}

Diffusion bonding is an attractive joining technique for many advanced materials, ${ }^{1)}$ particularly, when these are degraded by traditional fusion welding processes and when the need for environmental or thermal stability in thin-sheet structures precludes adhesive bonding. There is also the possibility of combining diffusion bonding with superplastic forming, SPF/DB, to manufacture complex sheet structures with reduced weight and fabrication costs compared to mechanically fastened structures. ${ }^{2,3)}$ Recently, the SPF/DB technique has been successfully applied to superplastictitanium alloys structures in the aerospace industry. ${ }^{4,5}$ Despite these advantages, there are a lot of factors, pressure, time and temperature, relating to diffusion bonding quality. In order to produce sound joining, it is important to examine the optimal diffusion bonding conditions such as pressure, time and temperature and so on. In the previous investigations, these optimal conditions have been obtained using superplastic-titanium alloys, ${ }^{4-7)}$-aluminum alloys, ${ }^{8,9)}$-magnesium alloys ${ }^{10-12)}$ and -steels. ${ }^{13,14)}$ However, it is very difficult to obtain its optimal conditions by experiments. To date, the authors proposed diffusion bonding model to predict the optimal relationship between the bonding time and pressure. ${ }^{10)}$ In previous report, theoretical prediction for attaining good bonding condition was in good agreement with experimental results.

In diffusion bonding, the oxide film is also big problem to obtain high quality joining. There have been reported several solution of this problem; inserted inter layer, ${ }^{15,16)}$ surface treatment, ${ }^{10-13,17-19)}$ carried out diffusion bonding in vacuum $^{5,6)}$ or inert gas such as Ar. ${ }^{9,20)}$ The surface treatment, especially grit blast, is the most famous method in this solution. Before diffusion bonding, grit blast treatment is performed to remove the surface oxides and supply rugged surface. Many researchers point out that this technique is effective to remove the surface oxides and obtains higher quality in diffusion bonding. It is assumed that this technique will be carried out more and more in the future. ${ }^{12,18,19)}$ On the other hand, we reported that the value of surface roughness created by surface treatment was important factor affecting diffusion bonding quality, ${ }^{10)}$ because of the difference of diffusion bonding process by the surface roughness size. However, based on the diffusion bonding process, the detail relationship between surface roughness and diffusion bonding quality has not been investigated yet. Therefore, in the present paper, by using superplastic materials, diffusion bonding tests were carried out under superplastic conditions by using theoretical estimation. We investigated the relationship between surface roughness and diffusion bonding quality. In addition, the optimal surface roughness was analyzed to obtain high quality joining.

\section{Experimental Procedure}

The materials used in the present study were sheets of $\mathrm{Mg}-$ Al-Zn alloy, AZ31, with different grain sizes of 11 and $28 \mu \mathrm{m}$. Two kinds of present used materials were obtained the superplastic behavior at temperature of $\sim 673 \mathrm{~K}$. The superplastic behavior is described in detail in the previous paper. ${ }^{10)}$ Before diffusion bonding, the grit blast treatments were performed to remove the surface oxides and supply rugged surface by using alumina particles. In order to create several kinds of rugged surface roughness, two kinds of alumina particles, diameter of 20 and $5 \mu \mathrm{m}$, were used in several separations between the specimen and the muzzle of a gun. After surface treated specimens were cleaned in ethanol using ultrasonic vibration cleaner, diffusion bonding tests were carried out at conditions (bonding temperature and bonding pressure) similar to those corresponding to the superplastic properties. From the result of tensile tests, ${ }^{10)}$ bonding temperature and pressure were $673 \mathrm{~K}$ and $4 \mathrm{MPa}$, respectively. In terms of bonding time, optical times were calculated based on the theoretical diffusion bonding model. The calculated results were listed in Table 1 . Table 1 also included the results of surface roughness measurement. Diffusion bonding quality was assessed by the compressive lap shear tests. The compressive lap shear tests are also described in detail in the previous paper. The microstructural observation also carried out in order to investigate the oxide film near the original surface by using Electron Probe X-ray Micro Analyzer (EPMA). 
Table 1 The relationship between the calculated results on optimal bonding time and the surface roughness in the present used materials.

\begin{tabular}{|c|c|c|c|}
\hline Grain size, $d / \mu \mathrm{m}$ & Alumina particle diameter $/ \mu \mathrm{m}$ & Surface roughness, $\lambda / \mu \mathrm{m}$ & Bonding time, $t / \mathrm{ks}$ \\
\hline \multirow{4}{*}{11} & as-received & 2.8 & $* * *$ \\
\hline & 5.0 & 4.5 & 0.6 \\
\hline & 20 & 25.4 & 3.3 \\
\hline & 20 & 30.4 & 4.5 \\
\hline \multirow{5}{*}{28} & as-received & 1.7 & $* * *$ \\
\hline & 5.0 & 4.5 & 0.6 \\
\hline & 5.0 & 9.5 & 4.3 \\
\hline & 20 & 24.5 & 16.3 \\
\hline & 20 & 30.7 & 21.8 \\
\hline
\end{tabular}

Table 2 The relationship between the ratio of compressive lap shear strength and the surface roughness after diffusion bonding test. Each of parent metal strengths, $\tau_{\mathrm{p}}$, was 89.1 (fine-grained) and $85.1 \mathrm{MPa}$ (coarse-grained), respectively.

\begin{tabular}{|c|c|c|c|c|}
\hline Grain size, $d / \mu \mathrm{m}$ & Surface roughness, $\lambda / \mu \mathrm{m}$ & Bondign time, $t / \mathrm{ks}$ & Lap shear strength, $\tau_{\mathrm{b}} / \tau_{\mathrm{p}} / \%$ & Mechanism \\
\hline \multirow{3}{*}{11} & 4.5 & 0.6 & 84.5 & $\mathrm{D}$ \\
\hline & 8.7 & 1.8 & 90.0 & $\mathrm{D}+\mathrm{P}$ \\
\hline & 30.4 & 4.5 & 89.5 & $\mathrm{D}+\mathrm{P}$ \\
\hline \multirow{4}{*}{28} & 4.5 & 0.6 & 82.1 & $\mathrm{D}$ \\
\hline & 9.5 & 4.3 & 83.7 & $\mathrm{D}$ \\
\hline & 25.4 & 16.3 & 92.4 & $\mathrm{D}+\mathrm{P}$ \\
\hline & 30.7 & 21.8 & 93.0 & $\mathrm{D}+\mathrm{P}$ \\
\hline
\end{tabular}

where $\mathrm{D}$ is diffusional controlled process and $\mathrm{P}$ is plasticity controlled process.

\section{Result and Discussion}

The relationship between compressive lap shear strength, $\tau_{\mathrm{b}} / \tau_{\mathrm{p}}$ where $\tau_{\mathrm{b}}$ is bonded lap shear strength and $\tau_{\mathrm{p}}$ is the parent metal lap shear strength, and surface roughness was listed in Table 2 . Since the ratio of $\tau_{\mathrm{b}} / \tau_{\mathrm{p}}$ could be obtained $\sim 0.8$ at all diffusion bonding testing conditions, the theoretical prediction conditions were in good agreement with experimental results. The maximum ratio of lap shear strength, $\tau_{\mathrm{b}} / \tau_{\mathrm{p}}$, was 0.93 at a bonding pressure of $4 \mathrm{MPa}$ for $21.8 \mathrm{ks}$. From Table 2 , the ratio of $\tau_{\mathrm{b}} / \tau_{\mathrm{p}}$ was independent of the values of surface roughness and grain sizes. This is resulted from the difference of diffusion bonding process by treated surface roughness and/or grain sizes.

When the treated surface specimen joined each other, the original separate surface form openings (designated herein, voids). In general, there are two void shrinkage processes: diffusional controlled process and plasticity controlled process. ${ }^{21,22)}$ However, the void shrinkage process is different with the value of treated surface roughness. Here, it has been generally reported that the intersection radius of diffusional controlled process and plastic controlled process, $r_{\mathrm{c}}$, would be given by: ${ }^{23)}$

$$
r_{\mathrm{c}} \approx\left(\frac{2 \Omega \delta D_{\mathrm{gb}}}{k T}\right)^{1 / 3}\left(\frac{\sigma}{\dot{\varepsilon}_{\text {creep }}}\right)^{1 / 3}
$$

where $\Omega$ is the atomic volume, $\delta$ is the grain boundary width, $D_{\mathrm{gb}}$ is the coefficient for grain boundary diffusion, $k$ is the Boltzmann's constant, $T$ is the temperature, and $\dot{\varepsilon}_{\text {creep }}$ is the power law equation. ${ }^{10,24)}$ When the treated surface roughness is smaller than the value of intersection radius, the void
Table 3 The material factor in magnesium alloys. ${ }^{25)}$

\begin{tabular}{lc}
\hline & $\mathrm{Mg}$ \\
\hline Atomic volume, $\Omega / \mathrm{m}^{3}$ & $2.33 \times 10^{-28}$ \\
Burger's vector, $b / \mathrm{m}$ & $3.21 \times 10^{-10}$ \\
Surface energy, $\gamma / \mathrm{Jm}^{-2}$ & $5.6 \times 10^{-1}$ \\
Grain boundary width, $\delta / \mathrm{m}$ & $6.42 \times 10^{-10}$ \\
Grain boundary diffusion, $D_{\mathrm{gb}} / \mathrm{m}^{2} \mathrm{~s}^{-1}$ & $7.8 \times 10^{-3} \exp (-Q / R T)$ \\
Activation energy, $Q / \mathrm{kJ} \mathrm{mol}^{-1}$ & 92 \\
\hline
\end{tabular}

shrinkage process is occurred diffusional controlled process during diffusion bonding. On the other hand, when the created surface roughness is larger than the intersection radius, the void shrinkage process is not only diffusional controlled process but also plasticity controlled process. Therefore, it is very important to understand the relationship between the intersection radius and surface roughness in order to discuss the void shrinkage process during diffusion bonding. By comparison of the value of intersection radius in eq. (1) and the treated surface roughness, the void shrinkage processes were also included in Table 2. The used materials factors were listed in Table 3 . $^{25}$ )

Based on this notion, the relationship between the ratio of $\tau_{\mathrm{b}} / \tau_{\mathrm{p}}$ and the value of $\lambda / d$, where $\lambda$ is the surface roughness, was shown in Fig. 1. This figure also included the previous data, ${ }^{10,11)}$ which were satisfied with experimental bonding times similar to theoretical bonding times at temperature of $673 \mathrm{~K}$. In Fig. 1, the void shrinkage process was both diffusional controlled process and plasticity controlled process at open symbol mark. The void shrinkage process was only diffusional controlled process at close symbol mark. 


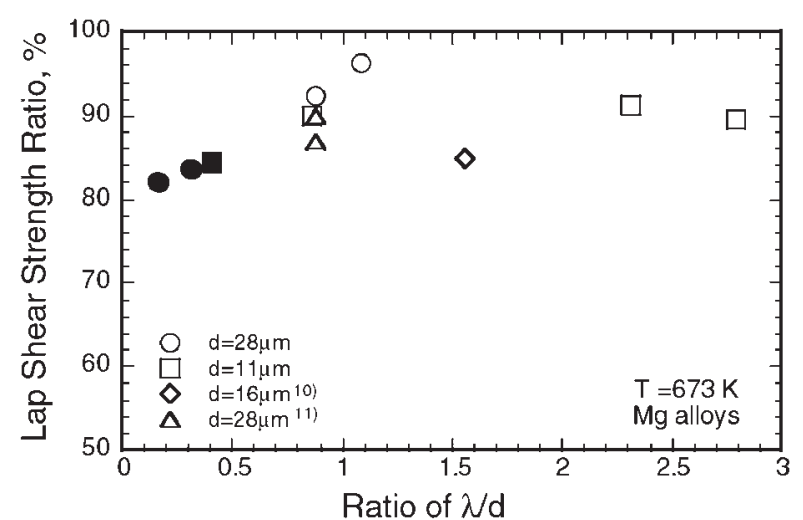

Fig. 1 The relationship between the ration of $\tau_{\mathrm{b}} / \tau_{\mathrm{p}}$ and the value of $\lambda / d$. The figure also includes the data for high quality joining at bonding times similar to theoretical results. ${ }^{10,11)}$

In case of low ratio of $\tau_{\mathrm{b}} / \tau_{\mathrm{p}}$, the void shrinkage process was only diffusional controlled process. On the other hand, the ratio of $\tau_{\mathrm{b}} / \tau_{\mathrm{p}}$ was high value at void shrinkage process of both diffusional controlled process and plasticity controlled process. From above results, it is found that the plasticity controlled process needed to obtain high quality joining. In general, since the diffusion bonding is carried out at elevated temperature, the oxide film is created on the interfacial surface. On superplastic-aluminum alloys and -magnesium alloys, this oxide film prevent the high quality joining. Recently, many researchers pointed out that the grain boundary sliding process break the surface oxide layers. ${ }^{26,27)}$ When the machined magnesium alloys chips were carried out the hot extrusion utilizing superplastic flow, the chipextruded materials attained good bonding and mechanical properties. Therefore, it is considered that the high quality joining was obtained at void shrinkage process including plasticity controlled process. In diffusion bonding, there is the affinity of grain boundary sliding, plasticity controlled process, and diffusion bonding quality.

From Fig. 1, it is also found that the ratio of $\tau_{\mathrm{b}} / \tau_{\mathrm{p}}$ increased up to $\lambda / d \approx 1$, afterward, maintained the constant. It is assumed that there are also the relationship between grain size and surface roughness in diffusion bonding quality. During diffusion bonding, it is noted that the plasticity controlled process would be mainly grain boundary sliding. The mechanism of grain boundary sliding would be grain rotation and/or movement. ${ }^{2,3)}$ The spacing, which create voids, is important to occur grain boundary sliding. Therefore, the optimal surface roughness would be equal to $\geq d$. However, from Table 1, the longer the bonding time is, the larger the surface roughness is. It is concluded that the optimal surface roughness would be similar to grain size.

The microstructural observation by using EPMA was shown in Fig. 2: (a) $\tau_{\mathrm{b}} / \tau_{\mathrm{p}}=0.90$ in $4.5 \mathrm{ks}$ and $(\mathrm{b}) \tau_{\mathrm{b}} / \tau_{\mathrm{p}}=$ 0.84 in $4.3 \mathrm{ks}$, respectively. The white arrows show the original surface. By comparison of Figs. 2(a) and (b), nevertheless the conditions, bonding times and pressures, were similar to each other, the distribution of oxygen near the original surface was different. There are a lot of distributed oxygen near the only original surface in Fig. 2(b), however, little oxygen distributed in Fig. 2(a). The difference of the distribution type was resulted from the void shrinkage process. From the data in Table 2, Fig. 2(a) was the both diffusional controlled process and plasticity controlled process, and Fig. 2(b) was only diffusional controlled process. Satoh et al. reported that the superplastic phenomena could be also achieved the homogeneous dispersion of fine ceramic powders within the matrix by using the compounding method. ${ }^{28)}$ In diffusion bonding, it is assumed that the oxide would be mixed into grain boundary by plasticity controlled process. On the other hand, form Fig. 2(b), it is apparent that the strong oxide film could not move easily by only diffusional controlled process. Therefore, since the oxide film on original surface prevented from high quality joining, the ratio of $\tau_{\mathrm{b}} / \tau_{\mathrm{p}}$ was about $\sim 0.8$.

In order to obtain high quality joining, (i) the void shrinkage process would be included not only diffusional controlled process but also plasticity controlled process, and (ii) the optimal value of surface roughness would be similar to grain sizes.

\section{Summary}

Based on the theoretical diffusion bonding model, the diffusion bonding behavior was investigated in superplastic AZ31 magnesium alloy. The following results were obtained.

(1) From diffusion bonding tests, the theoretical prediction conditions were in good agreement with experimental results.

(2) When the ratio of $\tau_{\mathrm{b}} / \tau_{\mathrm{p}}$ was obtained high value, the void shrinkage was not only plasticity controlled process but also diffusional controlled process during diffusion bonding. The plasticity controlled process was important process in high quality joining.

(3) In order to obtain high quality joining, the optimal surface roughness was the value of grain size.

\section{Acknowledgments}

The author is grateful to Dr. H. Watanabe (Osaka Municipal Technical Research Institute) for his surface roughness measurement. This work was supported by Grant-in-Aid for JSPS Fellows. A part of this work was also performed under the inter-university cooperative research program of the Institute for Materials Research, Tohoku University. 


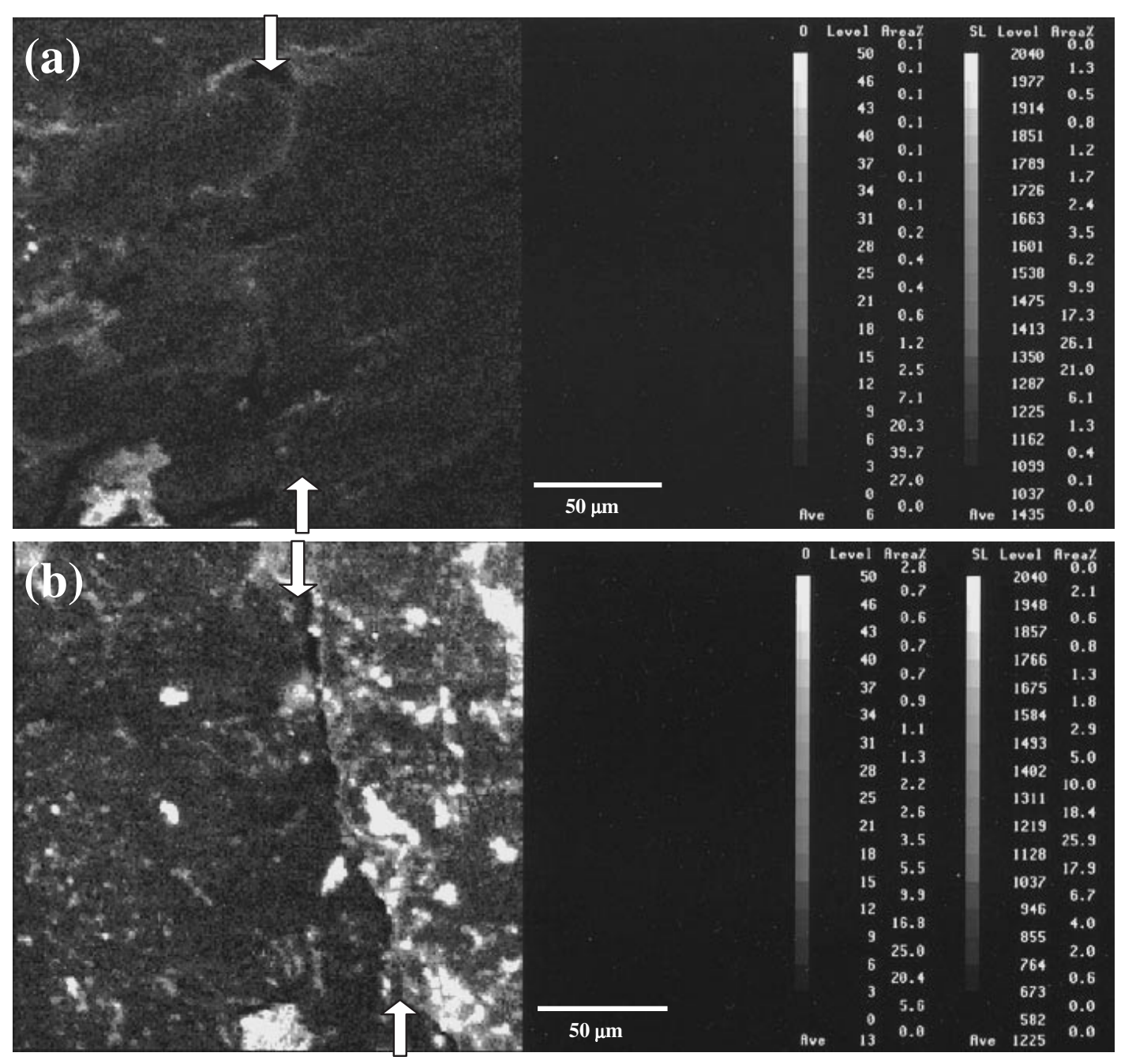

Fig. 2 The microstructural observation by using the EPMA: (a) $\tau_{\mathrm{b}} / \tau_{\mathrm{p}}=0.90$ in $4.5 \mathrm{ks}$ and (b) $\tau_{\mathrm{b}} / \tau_{\mathrm{p}}=0.84$ in $4.3 \mathrm{ks}$, respectively.

\section{REFERENCES}

1) P. G. Partridge and C. M. Ward-Close: Metals. Mater. 5 (1989) 334.

2) J. Pilling and N. Ridley: Superplasticity in Crystalline Solide, (Institute of Metals, 1980).

3) T. G. Nieh, J. Wadsworth and O. D. Sherby: Superplasticity in metals and ceramics, (Cambridge University Press, 1997).

4) K. Iizumi, A. Ogawa and N. Suzuki: J. Jpn. Inst. Light. Metals. 49 (1999) 368-372.

5) G. Cam, H. Clemens, R. Gerling and M. Kocak: Z. Metallk. 90 (1999) 284-288.

6) M. F. Islam, J. Pilling and N. Ridley: Mater. Sci. Tech. 13 (1997) 10451050.

7) M. T. Salehi, J. Pilling, N. Ridley and D. L. Hamilton: Mater. Sci. Eng. A150 (1992) 1-6.

8) J. Pilling and N. Ridley: Mater. Sci. Tech. 3 (1987) 353-359.

9) H. Y. Wu, S. Lee and J. Y. Wang: J. Mater. Proc. Tech. 75 (1998) 173 179.

10) H. Somekawa, H. Watanabe and K. Higashi: Mater. Trans. 44 (2003) 496-503.

11) H. Somekawa, H. Hosokawa, H. Watanabe and K. Higashi: Mater. Sci. Eng. A339 (2003) 328-333.

12) H. Somekawa, H. Watanabe, T. Mukai and K Higashi: Scr. Mater. 48 (2003) 1249-1254.

13) N. Ridley, M. T. Salehi and J. Pilling: Mater. Sci. Tech. 8 (1992) 791-
795.

14) M. F. Islam and N. Ridley: Scr. Mater. 38 (1998) 1187-1193.

15) A. Urena, J. M. Gomez de Salazar, J. Quinones and J. J. Martin: Scr. Mater. 34 (1996) 617-623.

16) P. G. Partridge and D. V. Dunford: J. Mater. Sci. 22 (1987) 1597-1608.

17) H. Somekawa, H. Hosokawa, H. Watanabe and K. Higashi: Mater. Trans. 42 (2001) 2075-2079.

18) J. Pilling: Mater. Sci. Eng. A100 (1988) 137-144.

19) A. S. Zuuzi, H. Li and G. Dong: Mater. Sci. Eng. A270 (1999) 244 248.

20) D. V. Dunford and P. G. Partridge: Mater. Sci. Tech. 8 (1992) 385-398.

21) J. W. Hancock: Metal. Sci. 10 (1976) 319-325.

22) M. V. Speight and W. Beere: Metal. Sci. 9 (1975) 190-191.

23) A. H. Chokshi and T. G. Langdon: Acta Metall. 35 (1987) 1089-1101.

24) H. Watanabe, T. Mukai, M. Kohzu, S. Tanabe and K. Higashi: Mater. Trans., JIM 40 (1999) 809-814.

25) H. J. Frost and M. F. Ashby: Deformation-mechanism Maps, (Pergamon Press, Oxford, 1982) pp. 44.

26) H. Watanabe, K. Moriwaki, T. Mukai T, K. Ishikawa, M. Kohzu and K. Higashi: J. Mater. Sci. 36 (2001) 5007-5010.

27) Y. Chino, R. Kishihara, K. Shimojima, H. Hosokawa, Y. Yamada, C. Wen, H. Iwasaki and M. Mabuchi: Mater. Trans. 43 (2002) 2437-2442.

28) M. Satoh, K. Higashi, F. Hamano, K. Miyanami, T. Ito and H. Yoshida: J. Mater. Sci. 27 (1992) 5003-5007. 